Relations industrielles

Industrial Relations

\title{
A Dictionary of Canadian Economics by David Crane, Edmonton, Hurtig Publishers, 1980, 372 pp.
}

\section{Gérard Dion}

Volume 35, numéro 2, 1980

URI : https://id.erudit.org/iderudit/029074ar

DOI : https://doi.org/10.7202/029074ar

Aller au sommaire du numéro

Éditeur(s)

Département des relations industrielles de l'Université Laval

ISSN

0034-379X (imprimé)

1703-8138 (numérique)

Découvrir la revue

Citer ce compte rendu

Dion, G. (1980). Compte rendu de [A Dictionary of Canadian Economics by David Crane, Edmonton, Hurtig Publishers, 1980, 372 pp.] Relations industrielles /

Industrial Relations, 35(2), 344-346. https://doi.org/10.7202/029074ar

Tous droits réservés ( Département des relations industrielles de l'Université Laval, 1980
Ce document est protégé par la loi sur le droit d'auteur. L'utilisation des services d'Érudit (y compris la reproduction) est assujettie à sa politique d'utilisation que vous pouvez consulter en ligne.

https://apropos.erudit.org/fr/usagers/politique-dutilisation/ 
de respecter certains principes fondamentaux (liberté syndicale et non-discrimination) et l'obligation de présenter des rapports au sujet des conventions non ratifiées et des recommandations. Le deuxième chapitre traite de la ratification des conventions et des obligations qui en découlent: l'obligation d'appliquer les conventions ratifiées et l'obligation de présenter annuellement des rapports sur les mesures prises pour en assurer l'application. L'auteur discute aussi à ce chapitre de l'importante question des rapports entre la norme internationale et la loi nationale (aux pages 234 et ss.). Les six derniers chapitres de cette partie traitent du contrôle de l'application des normes internationales du travail. L'auteur y expose les diverses méthodes qui ont assuré depuis 1919 un contrôle efficace et impartial des normes internationales du travail. Quant à l'objet du contrôle international, l'auteur note qu'à l'origine, l'objectif du système de contrôle consistait à assurer l'exécution des obligations résultant de la ratification des conventions par les Etats, mais qu'aujourd'hui il a été étendu à la promotion de la mise en oeuvre des normes internationales du travail même en l'absence de toute obligation (à la page 256).

Les normes internationales du travail ont reçu un très large écho au travers le monde. À ce sujet, l'auteur rappelle avec justesse (aux pages 244-245) que l'impact des normes internationales du travail dépasse largement le nombre des cas examinés par les différentes instances chargées du contrôle. Les normes internationales du travail constituent aujourd'hui un droit commun international auquel les Etats, développés ou en voie de développement, se réfèrent comme une source d'inspiration dans l'élaboration de la loi nationale.

Un index doublé d'une table des matières fort détaillée favorise une consultation rapide de l'ouvrage.

En dépit du fait que la littérature soit abondante en droit international du travail, nous devons saluer la publication d'une monographie comme celle que nous présente Nicolas Valticos. L'activité normative de l'O.I.T. et des autres organismes internationaux dans le secteur du travail est encore trop méconnue, chez nous comme ailleurs, pour que l'on puisse se dispenser d'un ouvrage introductif tel que International Labour Law.

Comme les écrits antérieurs de Nicolas Valticos, cette monographie s'appuie sur une vaste expérience résultat d'une longue carrière au sein du B.I.T., notamment, pendant plusieurs années, à titre de chef du Département des normes internationales du travail. Nous devons remercier l'auteur de nous la faire partager.

\section{Alain BARRÉ}

Université Laval

A Dictionary of Canadian Economics by David Crane, Edmonton, Hurtig Publishers, 1980, 372 pp.

L'économique joue un rôle important dans le monde d'aujourd'hui et n'intéresse pas seulement les spécialistes. À chaque jour, à la radio, à la télévision et dans les journaux pour présenter et expliquer les problèmes d'ordre économique, on emploie une multitude de termes et d'expressions techniques dont le sens véritable n'est pas toujours compris.

Jusqu'ici, les Canadiens étaient obligés de recourir à des dictionnaires en provenance des Etats-Unis ou de la Grande Bretagne qui, on le comprend facilement, n'avaient pas été préparés en tenant compte du contexte particulier dans lequel nous vivons au Canada.

Préoccupé par cette lacune, le directeur de la page économique du journal The Toronto Star, David Crane, a voulu la combler en publiant A Dictionary of Canadian Economics.

Présentées par ordre alphabétique, on y trouve 2500 entrées comprenant non seulement les termes les plus usuels utilisés en économique et quelques uns en relations in- 
dustrielles, mais encore des notices sur les principales institutions, organisations et agences gouvernementales tant fédérales que provinciales oeuvrant en ces domaines. Les choix que l'auteur a fait pour ces notices permet aux lecteurs d'obtenir rapidement des renseignements sur une multitude d'organisations dont ils entendent parler chaque jour et qu'ils ne pourraient se procurer sans avoir à effectuer des recherches longues et pénibles. Il est à remarquer que l'auteur ne s'est pas limité au Canada et aux provinces, mais qu'il a relevé les organismes internationaux qui affectent la vie économique et politique canadienne.

Malgré son titre, cet ouvrage est plus qu'un dictionnaire, c'est une sorte de mini encyclopédie. En effet, l'auteur, en bien des cas, ne se contente pas de livrer une simple définition des termes. Il y ajoute parfois un bref développement avec des explications et même des jugements.

En s'engageant dans cette voie légitime en vue de mieux servir ses lecteurs, l'auteur s'est ajouté une nouvelle difficulté à celles déjà énormes de produire un dictionnaire. Comme il faut être à la fois concis et bref, à quel moment doit-on s'arrêter pour ne pas être incomplet ou laisser le lecteur sous son appétit? Pourquoi, encore, accompagner une définition d'un développement et n'en pas faire pour une autre qui en mériterait pourtant autant?

Quelques exemples. Au mot Seniority, l'auteur parle de l'ancienneté privilégiée pour les chefs syndicaux (pourquoi pas pour d'autres catégories de travailleurs?) et laisse complètement de côté ce qui peut apparaître beaucoup plus important, la base de l'ancienneté: l'entreprise, l'établissement, le département, le métier, la tâche. Dans les multitudes des Rapports de Commission d'enquête ou d'étude sur diverses questions dont la présentation est extrêmement utile aux lecteurs, il mentionne le Rapport Freedman qui a eu son importance sur le point particulier, mais ne parle pas du Rapport Woods qui porte sur l'ensemble des relations du travail au Canada. Code of ethical practices est défini uniquement en fonction de celui du Congrès du travail du Canada alors qu'il en existe plusieurs autres. Il aurait peut-être pu inclure aussi Code national du bâtiment.

Si l'ensemble des définitions sont bien faites, certaines sont moins heureuses. Ainsi, il n'est pas juste de définir la grève comme " $a$ decision by employees in a bargaining unit to stop working, following the breakdown of collective bargaining and the failure of conciliation and mediation to produce an agreement with the employer on new collective agreement", car une grève peut survenir en d'autres circonstances comme l'auteur d'ailleurs le dit en parlant de "sympathy strike" dont la définition, elle aussi encore laisse à désirer.

Nous avons relevé encore dans certaines notices des inexactitudes. À International Confederation of Free Trade Unions "formed in 1949 by a number of national tradeunions congresses, including the Canadian Labour Congress", il aurait fallu dire Canadian Congress of Labour, car le premier n'a vu le jour qu'en 1956. Dire que l'American Federation of Labor-Congress of Industrial Organizations "is financed by per capita dues from member unions in Canada and the United States" va faire sursauter les membres canadiens des syndicats internationaux, car ils ne participent en aucune façon au financement de l'AFL-CIO, mais seulement à celui de leurs propres syndicats.

Enfin, l'auteur a eu la bonne idée de multiplier les corrélats, ce qui rend son ouvrages extrêmement utile. On constate cependant ici et là certaines omissions. Cost-of-living allowance clause renvoie à cola clause, mais cette définition ne se trouve nulle part.

Ces imperfections, qui sont minimes, si l'on considère l'ampleur de l'ouvrage et les difficultés inhérentes à sa réalisation, pourront être facilement corrigées dans une édition subséquente. Nous savons, par expérience, qu'une oeuvre semblable n'est jamais achevée. Comme tel, cet ouvrage est grandement valable et une première bien réussie. 
Il faut féliciter l'auteur d'avoir produit ce dictionnaire qui est un instrument indispensable aux hommes d'affaires, étudiants, journalistes, fonctionnaires et même au public en général et qui mérite la plus large diffusion possible.

\section{Gérard DION}

Université Laval

L'univers politique et syndiçal des cadres, par Gérard Grunberg et René Mouriaux, Paris, Presses de la Fondation Nationale des Sciences Politiques, 1979, 230 pp.

L'instrument de cette recherche est un sondage effectué en mai/juin 1974 auprès d'un échantillon de 1481 cadres de l'industrie et des services. Définie à partir du classement INSEE (ingénieurs et cadres administratifs supérieurs, techniciens et cadres administratifs moyens), la population étudiée s'organise en fonction de six variables: sexe, âge, qualification, taille des établissements, branches, régions.

En tenant compte du contexte de l'époque, de l'incidence de l'actualité sur les réponses - la datation, évitée dans la majorité des questions, est parfois utilisée comme repère - les auteurs ont voulu dégager les lignes de force qui sous-tendent les comportements des cadres. Leur propos n'était pas de fixer l'image définitive et complète de l'opinion des cadres au lendemain de l'élection de V. Giscard d'Estaing, mais d'en éclairer la cohésion et la dispersion, les déterminants et les tendances. C'est cette orientation de recherche, interrogeant les données de l'observation et les situant dans leur durée, qui valide les conclusions et les hypothèses présentées dans ce livre, à partir de matériaux rassemblés en 1974.

Avant d'aborder l'analyse des critères d'identité des cadres, les auteurs soulignent une première difficulté: la diversité des problématiques qui tentent de les situer. Procédant par larges regroupements, ils relèvent trois types d'approche, dont chacun est d'ailleurs loin d'être univoque.
La première fait appel à la notion de classe moyenne ou intermédiaire. D'Aristote à V. Giscard d'Estaing, une rétrospective rapide emprunterait à Guizot, Gambetta, Jaurès, la C.G.C., J. Royer, J. Chirac. Traditionnellement gratifiée de qualités de compétence et de mesure, la classe moyenne est vouée à un rôle médiateur. En ce sens, son influence ne peut que s'accroître, d'autant que l'évolution des conditions de vie entraîne, selon l'auteur de Démocratie française, «l'expansion d'un immense groupe central aux contours peu tranchés".

Les auteurs marxistes (C. Baudelot, R. Establet, N. Poulantzas) placent les cadres dans le champ d'affrontement des classes. Groupe fissuré par la bi-polarisation, traversé de courants contradictoires, d'idéologies qui le pénètrent de l'extérieur sans qu'il puisse, pour son propre compte, en sécréter aucune. Les cadres, dépourvus d'identité idéologique, n'ont pas d'avenir de classe.

Une troisième approche, selon A. Touraine, R. Dahrendorf, J.K. Galbraith, D. Bell, notamment, aussi différenciée que les précédentes, récuse la bi-polarisation marxiste en raison de l'évolution technique modifiant en profondeur les rapports de production et la structure sociale. La société postindustrielle multiplie les pouvoirs partiels et les intérêts spécifiques. Société de compromis et de turbulences, la compétence technique s'y affirme comme source de prééminence, rivale des légitimités instituées par la propriété et le pouvoir politique.

Chacune des thèses prend appui sur une délimination plus ou moins précise du groupe des cadres; toutes mettent en cause le classement INSEE en proposant d'autres coupes de population. G. Grunberg et R. Mouriaux ont cependant suivi l'INSEE pour explorer la relation entre les catégories 3 et 4 dont le rapprochement ne leur paraît pas neutre, mais aussi en raison du vaste terrain ainsi ouvert à l'influence des facteurs de cohésion et de fragmentation. Y a-t-il une conscience commune de spécificité d'intérêts reliant tous ceux qui appartiennent à cet ensemble? Les clivages qui le segmentent s'expriment-ils en 